\title{
Resonance Raman Optical Activity Shows Unusual Structural Sensitivity for Systems in Resonance with Multiple Excited States: Vitamin $\mathrm{B}_{12}$ Case
}

Ewa Machalska, Grzegorz Zajac, Anna Gruca, Fabio Zobi, Malgorzata Baranska, and Agnieszka Kaczor*

Cite This: J. Phys. Chem. Lett. 2020, 11, 5037-5043

Read Online

ABSTRACT: In this work, cobalamins with different upper axial substituents and a cobalamin derivative with a ring modification were studied using chiroptical spectroscopies, in particular resonance Raman optical activity (RROA), to shed light on the influence of structural modifications on RROA spectra in these strongly chiral systems in resonance with multiple excited states at $532 \mathrm{~nm}$ excitation. We have demonstrated that for these unique systems RROA possesses augmented structural specificity, surpassing resonance Raman spectroscopy and enabling at the same time measurement of cobalamins at fairy low concentrations of $\sim 10^{-5}$ mol dm${ }^{-3}$. The enhanced structural specificity of RROA is a result of bisignate spectra due to resonance via more than one electronic state. The observation of increased structural capability of RROA for cobalamins opens a new perspective for studying chiral properties of other biological systems incorporating d-metal ions.

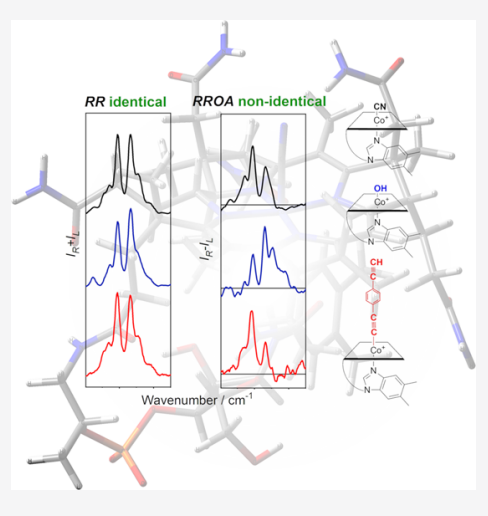

$\mathrm{R}$ aman optical activity (ROA) is a powerful structural probe of chirality for investigation of various forms of matter. The specific sensitivity of ROA spectroscopy to the chiral molecular environment makes it an efficient tool for studying biomolecules, e.g., proteins, amino acids, carbohydrates, or supramolecular structures. ${ }^{1}$ SCP-ROA (scattered circular polarization ROA) measures a small intensity difference in Raman scattering of right and left circularly polarized light from optically active molecules. ${ }^{2}$ Due to the fact that the ROA effect is a fairly weak phenomenon, the intensities of ROA signals are usually 3-4 orders of magnitude smaller than Raman signals; therefore, methods providing ROA signal enhancement have been attracting a considerable amount of attention. $^{3-8}$ One of these methods is resonance ROA, a chiroptical analogue of resonance Raman spectroscopy. A resonance ROA (RROA) spectrum, when derived from a single isolated electronic state, is monosignate and opposite in sign to the electronic circular dichroism (ECD) band of the resonant electronic transition and exhibits signals with the same relative intensities as those in the parent resonance Raman (RR) spectrum. ${ }^{9}$ In the simplest formulated theory of resonance ROA, i.e., described above as single-electronic state (SES) theory, where strong resonance with only one electronic state is considered, only the Albrecht A-term of resonance Raman scattering contributes to the obtained ROA. ${ }^{10}$ An extension of the SES theory is the two-electronic state (TES) theory, ${ }^{11}$ where the resonance ROA signal appears to be due to both A- and B-term mechanisms. As a result, bisignate RROA spectra are obtained due to resonance with multiple excited electronic states. ${ }^{12}$ Recently, ROA and other chiroptical spectroscopies in conjunction with quantumchemical calculations were applied to study the vibrational and electronic properties of chiral coordination complexes showing that the presence of these metal ions significantly increased the magnitude of the chiral signal due to resonance via a single electronic state or multiple electronic states. ${ }^{13-15}$

Vitamins $\mathrm{B}_{12}$ (cobalamins) are biologically active compounds incorporating a transition metal ion. These exogenous compounds (Scheme 1) have a unique structure based on a corrin ring encapsulating a low-spin $\mathrm{Co}^{3+}$ ion coordinated equatorially by four pyrrolic nitrogen atoms and two axial ligands. 5,6-Dimethylbenzimidazole is covalently bound to the ring in one of its axial coordination positions (lower), and the other (upper) can be occupied by various substituents such as cyano, hydroxyl, methyl, and adenosyl groups. ${ }^{16}$ Cobalamins play an essential role in various biological processes, such as nucleic acid metabolism and formation of red blood cells. ${ }^{17,18}$ Also, functionalized cobalamins, with a modified ring structure or upper axial substituent, have diverse biological properties; for example, they can be used as a scaffold for the delivery of antimalarial drugs to erythro- and hepatocytes. ${ }^{19}$

Received: April 21, 2020

Accepted: June 5, 2020

Published: June 5, 2020 
Scheme 1. Molecular Structures of Cobalamins with Different Upper Axial Substituents, Including Cyanocobalamin $(\mathrm{R}=\mathrm{CN})$, Hydroxocobalamin $(\mathrm{R}=\mathrm{OH}), \mathbf{1}, 4$-Diethynylbenzenecobalamin $(\mathrm{R}=\mathrm{C} \equiv \mathrm{C}-\mathrm{Ph}-\mathrm{C} \equiv \mathrm{CH})$, and a Cobalamin Ring Derivative $\left[\left(\mathrm{C}_{10}\right) \mathrm{H}=\mathrm{Br}\right]$

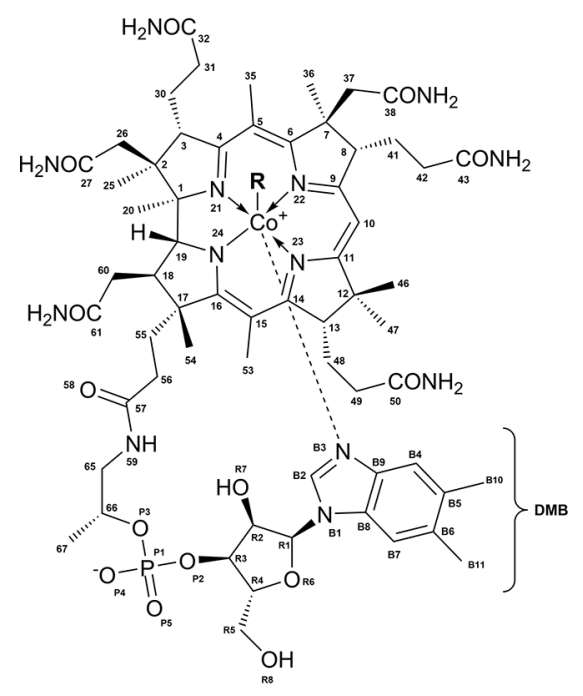

The corrin ligand exhibits a single helical sense of absolute $R$ configuration of its stereocenters. ${ }^{20}$ The structure of the macrocyclic corrin ring resembles the metalloporphyrin system, but with relevant differences. The most important is the presence of the additional side chains and the more reduced character of the corrin ring compared with that of the porphyrin one, which provides a greater degree of conformational freedom of corrinoids. It was found that the increased flexibility of the corrin macrocycle plays a particular role in $\mathrm{Co}-\mathrm{C}$ bond activation by $\mathrm{B}_{12}$-dependent enzymes. ${ }^{21-23}$

Ultraviolet-visible (UV-vis), ECD, and Raman spectroscopy were applied to study structures of several corrinoids in aqueous and organic solvents. ${ }^{24,25}$ Strong absorption bands in the visible and near-UV region, due to $\pi-\pi^{*}$ electronic transitions, cause significant resonance enhancement in the Raman spectra under visible light excitation. A few previous works demonstrated that $R R$ spectra of vitamin $B_{12}$ and its various analogues are strikingly similar, i.e., independent of the nature of the upper axial substituent. ${ }^{24}$ The similarity of the Raman spectra is not fully reflected in the similarity of the absorption UV-vis spectra showing that the excited states of the various derivatives of $\mathrm{B}_{12}$ do differ. ${ }^{24}$ On the contrary, ECD spectroscopy was shown to be more sensitive for replacement of an axial ligand of corrinoids, although due to the nature of the method (few broad features in the spectra) a detailed analysis of the structure using ECD is unavailable. It was confirmed that distinct changes in the ECD and UV-vis spectra were not related to chemical modifications of the corrin ring, but could result from electronic or conformational effects. 25

Intense ECD spectra of cobalamins in the UV-vis range were previously reported showing that cobalamins' ECD signals were quite sensitive to the substituent in the upper axial position. ${ }^{25}$ Our study demonstrates (Figure 1) that significant spectral differences in the ECD spectra are observed when the atom linking the $\mathrm{Co}$ ion is different ( $\mathrm{CNCbl}$ vs $\mathrm{OHCbl}$ spectra), although some differences are also noticed between $\mathrm{CNCbl}$ and $\mathrm{HC} \equiv \mathrm{C}-\mathrm{Ph}-\mathrm{C} \equiv \mathrm{CCbl}$, where the cyano


$\mathrm{HC} \equiv \mathrm{C}-\mathrm{Ph}-\mathrm{C} \equiv \mathrm{C} \mathrm{Cbl}$

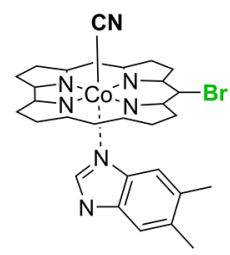

$\mathrm{CNCbl-Br}$

group is modified by a bulky $\mathrm{HC} \equiv \mathrm{C}-\mathrm{Ph}-\mathrm{C} \equiv \mathrm{C}-$ group. On the contrary, for a studied derivative with the ring modification, i.e., $\mathrm{CNCbl}-\mathrm{Br}$, the electronic spectra have characteristics similar to those of the spectra of its nonmodified counterpart, CNCbl. A detailed consideration of the assignment of the UV-vis and ECD spectra is presented in the Supporting Information.

As cobalamins have multiple electronic transitions in the vicinity of the ROA laser excitation at $532 \mathrm{~nm}$ (green line in Figure 1), we applied this method to study for the first time vibrational chiroptical properties of cobalamin derivatives. RR and RROA spectra of studied cobalamins recorded using an excitation line of $532 \mathrm{~nm}$ are presented in Figure 2.

In agreement with previously published data, ${ }^{26} \mathrm{RR}$ spectra of cobalamins with various upper axial substituents are strikingly similar and reflect the structure of the macrocycle due to the dominant contribution of the significantly resonantly enhanced ring vibrations. Practically all intense $R R$ bands have contributions from the corrin ring as demonstrated by the comparison of dicyanocobinamide RR spectrum (lacking a bulky DMB group) with the spectra of cobalamins ${ }^{27}$ and what is reflected in our quantum-chemical calculations (Supporting Information). Slight differences in the relative intensities and wavenumbers of the signals of all studied cobalamins result most probably from different resonance conditions and molecular (electronic) structure, respectively.

On the contrary, RROA signatures of analyzed cobalamins exhibit significantly more pronounced variations in the intensities of bands compared to RR spectra, which is clearly observed if the spectra are carefully investigated in the relevant spectral ranges (Figure 2, insets). According to a detailed analysis by Stich et al. ${ }^{25}$ and our quantum-chemical calculations (Supporting Information), there are at least three electronic states that might contribute to the resonance using $532 \mathrm{~nm}$ excitation for the considered cobalamins. In the case of $\mathrm{CNCbl}$ and $\mathrm{OHCbl}$, there are two negative electronic states and one positive electronic state in the vicinity of the excitation laser (Figure 1). An important consequence of this 

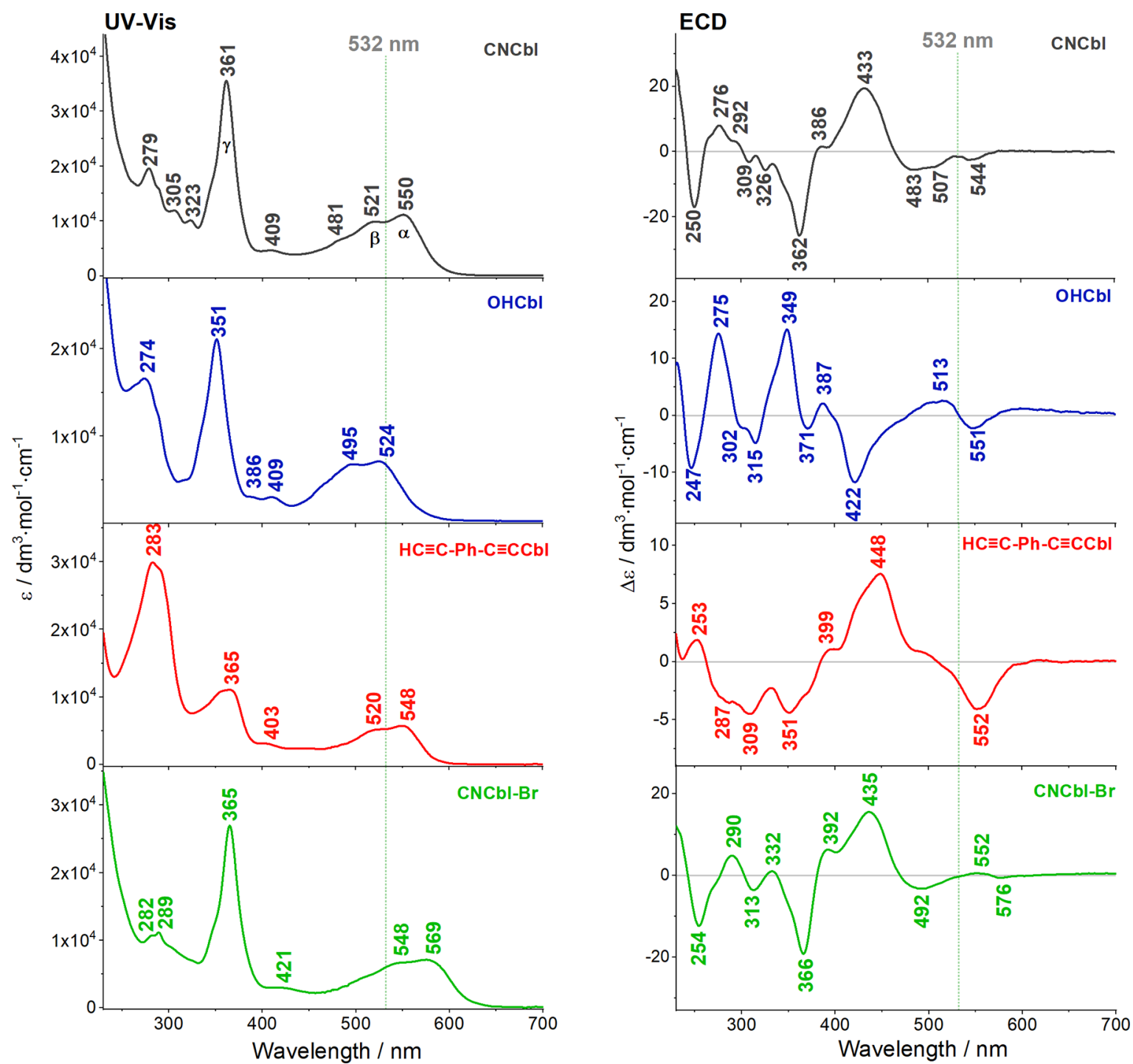

Figure 1. UV-vis and ECD spectra of cobalamins with different upper axial substituents: cyanocobalamin $(\mathrm{R}=\mathrm{CN})$, hydroxocobalamin $(\mathrm{R}=$ $\mathrm{OH})$, 1,4-diethynylbenzenecobalamin $(\mathrm{R}=\mathrm{C} \equiv \mathrm{C}-\mathrm{Ph}-\mathrm{C} \equiv \mathrm{CH})$, and a cobalamin ring derivative $\left[\left(\mathrm{C}_{10}\right) \mathrm{H}=\mathrm{Br}\right]$.

fact is that RROA spectra, although definitely recorded under the resonance conditions, are bisignate. This brings us to one of the most meaningful observations in this work. Our data demonstrate that differences in the electronic structure due to the different substituents scarcely affect RR spectra but result in the modification of resonance ROA conditions and bring significantly more pronounced variations in RROA intensities. This means that in the studied case, the sensitivity of RROA to subtle changes in the molecular structure is increased compared to that of RR spectroscopy. To the best of our knowledge, this is the first observation demonstrating augmented structural sensitivity of RROA compared to RR spectroscopy. For cobalamins with different upper axial substituents, it originates from a highly chiral chromophore for which resonance enhancement takes place via multiple electronic states that differ in energy and a sign of the rotatory strengths. In the case of CNCbl, RROA signals are mostly positive due to the proximity of the laser excitation to the negative ECD electronic transitions (first, $544 \mathrm{~nm}$, second, 507 $\mathrm{nm}$, and $483 \mathrm{~nm}$ band that is assigned to the vibrational progression of the first electronic transition) and slightly negative due to the third positive ECD transition at $433 \mathrm{~nm}$.
For $\mathrm{OHCbl}$, surprisingly, the proximity of both negative and positive ECD bands gives a similar, predominantly positive RROA spectrum. It demonstrates that the negative ECD (551 $\mathrm{nm}$ ) electronic state with the dominant corrinoid $\operatorname{ring} \pi \rightarrow \pi^{*}$ transition (HOMO $\rightarrow$ LUMO) has the most pronounced influence on the RROA spectra. This is contrary to the most recent RROA study of Sgammato et al. ${ }^{7}$ in which even slight changes in the intensity and energy of ECD bands resulted in the pronounced changes in RROA intensities. Additionally, Sgammato et al. ${ }^{7}$ reported a case in which RROA and RR are highly sensitive to a different axial ligand of heme. In our study, we highlight increased RROA specificity compared to RR in systems, where different axial ligands do not affect the spin state of the cobalt ion. According to our quantum-chemical calculations, molecular orbitals involved in the second (negative ECD) and third (positive ECD) electronic transitions of $\mathrm{CNCbl}$ are composed of not only the corrinoid ring $\pi$ orbitals but also $\mathrm{CN}-\pi$, DMB- $\pi$, and Co $3 \mathrm{~d}$ orbitals. The influence of that positive ECD transition on the sign of RROA is clearly seen in the experimental RROA spectrum of $\mathrm{CNCbl}$ and confirmed by the calculations, where the most negative feature located in the experimental spectra at $497 \mathrm{~cm}^{-1}$ is 

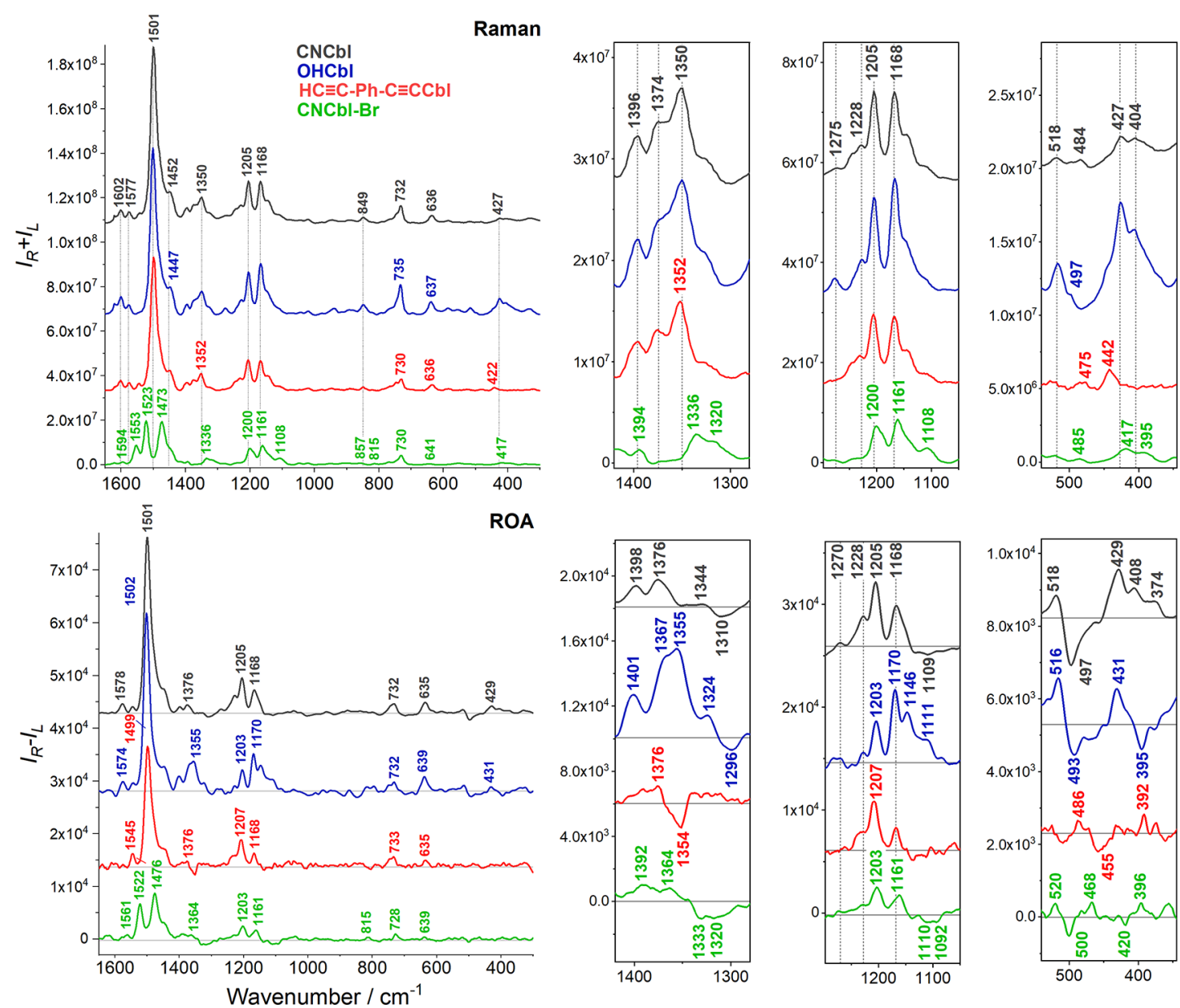

Figure 2. Raman and RROA spectra of cobalamins with different upper axial substituents: cyanocobalamin $(\mathrm{R}=\mathrm{CN})$, hydroxocobalamin $(\mathrm{R}=$ $\mathrm{OH})$, 1,4-diethynylbenzenecobalamin $(\mathrm{R}=\mathrm{C} \equiv \mathrm{C}-\mathrm{Ph}-\mathrm{C} \equiv \mathrm{CH})$, and a cobalamin ring derivative $\left[\left(\mathrm{C}_{10}\right) \mathrm{H}=\mathrm{Br}\right]$.

related mostly to the $\mathrm{Co}-\mathrm{C} \equiv \mathrm{N}$ bending, $\mathrm{C} \equiv \mathrm{N}$ twisting, and $\mathrm{DMB}$ ring breathing. The influence of the excitation of various electronic states on the RROA spectrum is clearly visible in the comparison of RROA spectra calculated using different excitation lines (Figure 3).

The closer the excitation line is to the first electronic state (443 nm, negative ECD), the more monosignate (positive) ROA is. For excitations at 400 and $420 \mathrm{~nm}$, bisignate ROA is observed due to proximity of two electronic transitions ( 2 and 3 ) that have opposite sign rotatory strengths. Far from resonance (650 $\mathrm{nm}$ excitation line), $\mathrm{CNCbl}$ also gives the bisignate ROA spectrum.

A closer look at the recorded spectra demonstrates that alterations in the relative intensities of the RROA bands are observed for all studied cobalamins with different upper axial substituents. $\mathrm{CNCbl}$ and $\mathrm{HC} \equiv \mathrm{C}-\mathrm{Ph}-\mathrm{C} \equiv \mathrm{CCbl}$ signals at $\sim 1500$ and $\sim 1205 \mathrm{~cm}^{-1}$, respectively, assigned to ring vibrations have increased relative intensity in the spectra compared to those of OHCbl.

On the contrary, the RROA spectrum of OHCbl shows an increased relative intensity of several positive bands due to the umbrella $\mathrm{CH}_{3}$ bending vibrations in the range of 1300-1400 $\mathrm{cm}^{-1}$ along with the signals in the range of $1100-1190 \mathrm{~cm}^{-1}$, assigned mostly to the $\mathrm{CH}_{2}$ twisting and $\mathrm{C}-\mathrm{N}$ and $\mathrm{C}-\mathrm{C}$ stretching modes. In the $\mathrm{CNCbl}$ and $\mathrm{HC} \equiv \mathrm{C}-\mathrm{Ph}-\mathrm{C} \equiv \mathrm{CCbl}$ RROA spectra, signals in the aforementioned ranges are considerably less intense or have the opposite intensity. In particular, in the $\mathrm{HC} \equiv \mathrm{C}-\mathrm{Ph}-\mathrm{C} \equiv \mathrm{CCbl}$ spectrum, a negative band is observed at $1354 \mathrm{~cm}^{-1}$ with the shoulder at $\sim 1370$ $\mathrm{cm}^{-1}$, opposite in sign to signals observed in the OHCbl spectrum. Negative RROA intensity is also clearly observed in the range of $300-600 \mathrm{~cm}^{-1}$, where mostly bands associated with the $\mathrm{Co}-\mathrm{R}$ vibrations are predicted. In addition, this range confirms that recorded RROA spectra of cobalamins with different upper axial substituents are markedly different. Calculated Raman and ROA spectra of CNCbl (Supporting Information) are in good agreement with experimental results and clearly confirm that the spectra are bisignate.

We investigated and characterized also a cobalamin with a modified ring structure (Scheme 1), where the hydrogen atom linked to $\mathrm{C}_{10}$ was replaced with a bromine atom, to illustrate the impact of a ring modification on RROA (Figure 2) spectra of cobalamin.

Comparison of the $\mathrm{CNCbl}$ and $\mathrm{CNCbl}-\mathrm{Br}$ spectra shows that RR signatures are markedly affected by the ring modification (Figure 2) due to different resonance conditions [the $\alpha / \beta$ absorption band due to the $\pi \rightarrow \pi^{*}$ transition along the long $\mathrm{C}_{5}-\mathrm{C}_{15}$ axis is red-shifted approximately $20-30 \mathrm{~nm}$ in $\mathrm{CNCbl}-\mathrm{Br}$ relative to $\mathrm{CNCbl}$ (Figure 1)] caused by the altered electronic/geometric structure of the corrin ring resulting from $\mathrm{Br}$ substitution. Due to the red-shift of the $\alpha / \beta$ absorption band in $\mathrm{CNCbl-Br}$, resonance conditions are altered compared to those of $\mathrm{CNCbl}$ and negative bands are observed in the $\mathrm{CNCbl-Br}$ RROA spectrum in various ranges. A characteristic 

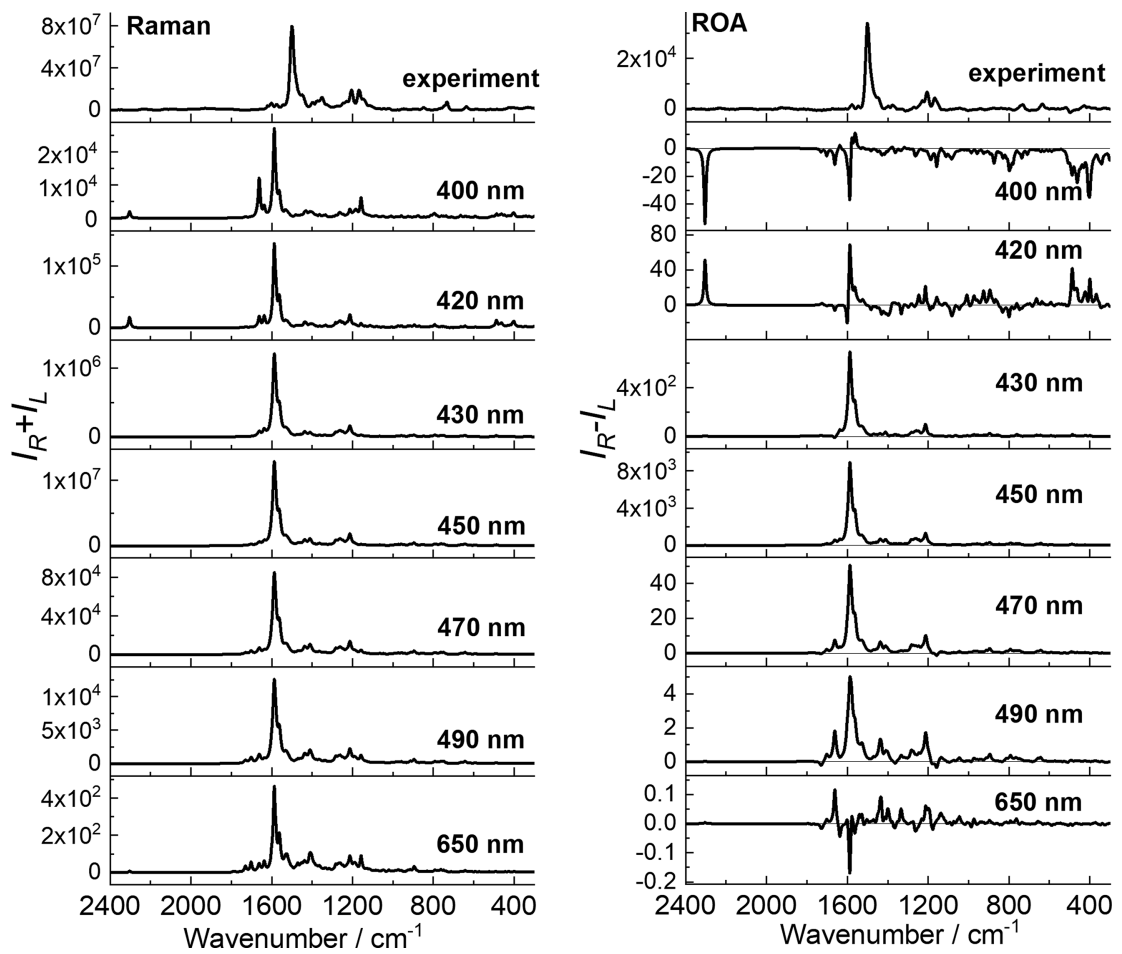

Figure 3. Comparison of experimental and calculated pre-resonance Raman and ROA spectra of CNCbl calculated at the CAM-B3LYP/6-31G(d)/ MDF10/6-31G(d)/PCM level of theory, using 400, 420, 430, 450, 470, 490, and $650 \mathrm{~nm}$ excitation lines.

RROA feature of CNCbl-Br is also the band at $815 \mathrm{~cm}^{-1}$ [assigned to the nearly isolated $\mathrm{C}-\mathrm{C}_{10}-\mathrm{C}(-\mathrm{H})$ vibrations (Supporting Information)] absent from RR and RROA spectra of other corrinoids. The intensity of this band increases due to substitution with a heavy atom, i.e., a bromine atom. Although ring modifications affect both RR and RROA spectra, for a studied cyanocobalamin with a ring modification, RROA is still a better probe of the molecular structure.

The obtained RROA/RR (CID) and ECD/UV-vis ( $g$ factor) ratios mostly do not obey the SES theory ${ }^{9}$ relation, $\mathrm{CID}=-1 / 2 g$, which is not surprising in the multiple excited state RROA. However, CIDs preserve the same order of magnitude and opposite signs as the $g$-factors of related resonance transitions discussed above (Supporting Information). For CNCbl-Br, the CIDs of a majority of positive RROA bands equal approximately $10^{-4}$, while related negative ECD transitions at 544,507 , and $483 \mathrm{~nm}$ possess $g$-factors close to $-10^{-4}$. A similar situation is observed for the negative RROA band at $497 \mathrm{~cm}^{-1}$, where the CID value and the $g$-factor of positive ECD at $433 \mathrm{~nm}$ are $-4 \times 10^{-3}$ and $5 \times 10^{-3}$, respectively. Furthermore, the most intense positive RROA band of $\mathrm{CNCbl}\left(1501 \mathrm{~cm}^{-1}\right)$ possesses a CID of $4.3 \times 10^{-4}$, which is almost twice as high as the absolute value of the $g$ factor at $544 \mathrm{~nm}\left(-2.4 \times 10^{-4}\right)$ and 9 times higher than the absolute value of the $g$-factor at $507 \mathrm{~nm}\left(-0.5 \times 10^{-4}\right)$, but it is surprisingly less than half of the $g$-factor at $483 \mathrm{~nm}(-8.5 \times$ $\left.10^{-4}\right)$. For other studied cobalamins, the CID and $g$-factor values show analogous behavior.

Although ECD is a sensitive tool for studying molecules, it is inherently limited to chromophores and as such does not provide detailed or local information about the molecular structure. VCD and ROA are significantly more informative in this context as they refer to well-localized vibrational modes, but they are characterized by low sensitivity that practically excludes their application in the study of biological samples.
RROA provides significant enhancement of the signal and could occur as an interesting alternative for ECD for the study of the molecular properties of biologically relevant systems. Nevertheless, in line with the single-electronic state theory of RROA, ${ }^{9}$ when the resonance occurs via a single electronic state, monosignate RROA spectra are either identical to the respective RR spectra or mirror images of the RR signatures. It is clear that in such cases RROA spectra do not provide a variety of structural information, apart from possibly distinguishing between optical isomers and measuring analytes at low concentrations.

In general, bisignate RROA spectra may be a result of the conformational freedom of the molecule, ${ }^{28}$ weak enhancement, and the presence of nonresonance bands along with the resonantly enhanced ones ${ }^{6}$ or excitation via more than one electronic state. ${ }^{11}$ Recently, it has been proven that bisignate RROA spectra can also be reproduced using a full quantum mechanical methodology, considering all RROA terms, along with Franck-Condon and Herzberg-Teller mechanisms. ${ }^{29}$ For cobalamins, bisignate RROA spectra result from the proximity of more than one electronic transition to the excitation wavelength, in agreement with previous detailed analysis of ECD spectra, $^{25}$ which was also confirmed by significant differences between RROA and RR spectra based on various relative intensities of the bands and not only the sign of the spectrum. Our observation shows that for strongly chiral systems in resonance with multiple excited states, RROA could be a method of augmented structural specificity, surpassing RR spectroscopy and at the same time enabling measurements of concentrations as low as $10^{-5} \mathrm{~mol} \mathrm{dm}^{-3}$. This finding opens a new perspective for studying chiral properties of biological systems incorporating d-metal ions. 


\section{ASSOCIATED CONTENT}

\section{(1) Supporting Information}

The Supporting Information is available free of charge at https://pubs.acs.org/doi/10.1021/acs.jpclett.0c01218.

Description of materials and methods, detailed description of quantum-chemical calculations, and detailed analysis of the spectra (PDF)

\section{AUTHOR INFORMATION}

\section{Corresponding Author}

Agnieszka Kaczor - Faculty of Chemistry and Jagiellonian Centre for Experimental Therapeutics (JCET), Jagiellonian University, Krakow 30-387, Poland; orcid.org/0000-00018337-8567; Email: agnieszka.kaczor@uj.edu.pl

\section{Authors}

Ewa Machalska - Faculty of Chemistry and Jagiellonian Centre for Experimental Therapeutics (JCET), Jagiellonian University, Krakow 30-387, Poland

Grzegorz Zajac - Jagiellonian Centre for Experimental Therapeutics (JCET), Jagiellonian University, Krakow 30-348,



Anna Gruca - Faculty of Chemistry and Jagiellonian Centre for Experimental Therapeutics (JCET), Jagiellonian University, Krakow 30-387, Poland

Fabio Zobi - Department of Chemistry, University of Fribourg, 1700 Fribourg, Switzerland

Malgorzata Baranska - Faculty of Chemistry and Jagiellonian Centre for Experimental Therapeutics (JCET), Jagiellonian University, Krakow 30-387, Poland

Complete contact information is available at:

https://pubs.acs.org/10.1021/acs.jpclett.0c01218

\section{Notes}

The authors declare no competing financial interest.

\section{ACKNOWLEDGMENTS}

This work was supported by the National Science Centre Poland (Projects 2017/25/B/ST4/00854 to A.K. and 2019/ 33/N/ST4/01986 to E.M.). This research was supported in part by PL-Grid Infrastructure.

\section{REFERENCES}

(1) Barron, L. D.; Hecht, L.; McColl, I. H.; Blanch, E. W. Raman Optical Activity Comes of Age. Mol. Phys. 2004, 102 (8), 731-744.

(2) Barron, L. D.; Bogaard, M. P.; Buckingham, A. D. Raman Scattering of Circularly Polarized Light by Optically Active Molecules. J. Am. Chem. Soc. 1973, 95 (2), 603-605.

(3) Vargek, M.; Freedman, T. B.; Lee, E.; Nafie, L. A. Experimental Observation of Resonance Raman Optical Activity. Chem. Phys. Lett. 1998, 287 (3-4), 359-364.

(4) Zajac, G.; Kaczor, A.; Pallares Zazo, A.; Mlynarski, J.; Dudek, M.; Baranska, M. Aggregation-Induced Resonance Raman Optical Activity (AIRROA): A New Mechanism for Chirality Enhancement. J. Phys. Chem. B 2016, 120 (17), 4028-4033.

(5) Dudek, M.; Machalska, E.; Oleszkiewicz, T.; Grzebelus, E.; Baranski, R.; Szcześniak, P.; Mlynarski, J.; Zajac, G.; Kaczor, A.; Baranska, M. Chiral Amplification in Nature: Studying Cell-Extracted Chiral Carotenoid Microcrystals via the Resonance Raman Optical Activity of Model Systems. Angew. Chem., Int. Ed. 2019, 58 (25), 8383-8388.

(6) Bogaerts, J.; Johannessen, C. On/off Resonance Raman Optical Activity of Human Serum Transferrin. J. Raman Spectrosc. 2019, 50 (5), 641-646.
(7) Sgammato, R.; Herrebout, W.; Johannessen, C. Resonance Raman Optical Activity of the imidazole-Myoglobin Complex: Titrating Enhancement. J. Raman Spectrosc. 2019, 50 (12), 19051913.

(8) Haraguchi, S.; Hara, M.; Shingae, T.; Kumauchi, M.; Hoff, W. D.; Unno, M. Experimental Detection of the Intrinsic Difference in Raman Optical Activity of a Photoreceptor Protein under Preresonance and Resonance Conditions. Angew. Chem., Int. Ed. 2015, 54 (39), 11555-11558.

(9) Nafie, L. A. Theory of Resonance Raman Optical Activity: The Single Electronic State Limit. Chem. Phys. 1996, 205 (3), 309-322.

(10) Champion, P. M.; Albrecht, A. C. Resonance Raman Scattering: The Multimode Problem and Transform Methods. Annu. Rev. Phys. Chem. 1982, 33 (1), 353-376.

(11) Merten, C.; Li, H.; Nafie, L. A. Simultaneous Resonance Raman Optical Activity Involving Two Electronic States. J. Phys. Chem. A 2012, 116 (27), 7329-7336.

(12) Luber, S.; Neugebauer, J.; Reiher, M. Enhancement and deEnhancement Effects in Vibrational Resonance Raman Optical Activity. J. Chem. Phys. 2010, 132 (4), 044113.

(13) Brichtová, E.; Hudecová, J.; Vršková, N.; Šebestík, J.; Bouř, P.; $\mathrm{Wu}, \mathrm{T}$. Binding of Lanthanide Complexes to Histidine-Containing Peptides Probed by Raman Optical Activity Spectroscopy. Chem. Eur. J. 2018, 24 (34), 8664-8669.

(14) Autschbach, J.; Jorge, F. E.; Ziegler, T. Density Functional Calculations on Electronic Circular Dichroism Spectra of Chiral Transition Metal Complexes. Inorg. Chem. 2003, 42 (9), 2867-2877.

(15) Le Guennic, B.; Hieringer, W.; Görling, A.; Autschbach, J. Density Functional Calculation of the Electronic Circular Dichroism Spectra of the Transition Metal Complexes $\left[\mathrm{M}(\text { phen })_{3}\right]^{2+}(\mathrm{M}=\mathrm{Fe}$, Ru, Os). J. Phys. Chem. A 2005, 109 (21), 4836-4846.

(16) Proinsias, K.; Giedyk, M.; Gryko, D. Vitamin $B_{12}$ : Chemical Modifications. Chem. Soc. Rev. 2013, 42 (16), 6605-6619.

(17) Fenech, M. Folate (Vitamin $B_{9}$ ) and Vitamin $B_{12}$ and Their Function in the Maintenance of Nuclear and Mitochondrial Genome Integrity. Mutat. Res., Fundam. Mol. Mech. Mutagen. 2012, 733 (1-2), $21-33$.

(18) Wagner, C. Biochemical Role of Folate in Cellular Metabolism. Clin. Res. Regul. Aff. 2001, 18 (3), 161-180.

(19) Rossier, J.; Nasiri Sovari, S.; Pavic, A.; Vojnovic, S.; Stringer, T.; Bättig, S.; Smith, S. G.; Nikodinovic-Runic, J.; Zobi, F. Antiplasmodial Activity and In Vivo Bio-Distribution of Chloroquine Molecules Released with a 4-(4-Ethynylphenyl)-Triazole Moiety from Organometallo-Cobalamins. Molecules 2019, 24 (12), 2310

(20) Senge, M. O.; Ryan, A. A.; Letchford, K. A.; MacGowan, S. A.; Mielke, T. Chlorophylls, Symmetry, Chirality, and Photosynthesis. Symmetry 2014, 6 (3), 781-843.

(21) Chowdhury, S.; Banerjee, R. Thermodynamic and Kinetic Characterization of Co-C Bond Homolysis Catalyzed by Coenzyme $\mathrm{B}_{12}$-Dependent Methylmalonyl-CoA Mutase. Biochemistry 2000, 39 (27), 7998-8006.

(22) Mebs, S.; Henn, J.; Dittrich, B.; Paulmann, C.; Luger, P. Electron Densities of Three $B_{12}$ Vitamins. J. Phys. Chem. A 2009, 113 (29), 8366-8378.

(23) Jensen, K. P.; Sauer, S. P. A.; Liljefors, T.; Norrby, P. O. Theoretical Investigation of Steric and Electronic Effects in Coenzyme $B_{12}$ Models. Organometallics 2001, 20 (3), 550-556.

(24) Stich, T. A.; Buan, N. R.; Brunold, T. C. Spectroscopic and Computational Studies of $\mathrm{Co}^{2+}$ corrinoids: Spectral and Electronic Properties of the Biologically Relevant Base-on and Base-off Forms of $\mathrm{Co}^{2+}$ cobalamin. J. Am. Chem. Soc. 2004, 126 (31), 9735-9749.

(25) Stich, T. A.; Brooks, A. J.; Buan, N. R.; Brunold, T. C. Spectroscopic and Computational Studies of $\mathrm{Co}^{3+}$-Corrinoids: Spectral and Electronic Properties of the $\mathrm{B}_{12}$ Cofactors and Biologically Relevant Precursors. J. Am. Chem. Soc. 2003, 125 (19), 5897-5914.

(26) Park, K.; Brunold, T. C. Combined Spectroscopic and Computational Analysis of the Vibrational Properties of Vitamin $B_{12}$ 
in Its $\mathrm{Co}^{3+}, \mathrm{Co}^{2+}$, and $\mathrm{Co}^{1+}$ Oxidation States. J. Phys. Chem. B 2013, 117 (18), 5397-5410.

(27) Mayer, E.; Gardiner, D. J.; Hester, R. E. Resonance Raman Spectra of Vitamin $\mathrm{B}_{12}$ and Dicyanocobalamin. Biochim. Biophys. Acta, Gen. Subj. 1973, 297 (2), 568-570.

(28) Zajac, G.; Kaczor, A.; Chruszcz-Lipska, K.; Dobrowolski, J. C.; Baranska, M. Bisignate Resonance Raman Optical Activity: A Pseudo Breakdown of the Single Electronic State Model of RROA? J. Raman Spectrosc. 2014, 45 (10), 859-862.

(29) Vidal, L. N.; Giovannini, T.; Cappelli, C. Can the Resonance Raman Optical Activity Spectrum Display Sign Alternation? J. Phys. Chem. Lett. 2016, 7 (18), 3585-3590. 